\title{
The EphB4 receptor promotes the growth of melanoma cells expressing the ephrin-B2 ligand
}

\author{
Nai-Ying Yang ${ }^{1,2, \dagger}$, Pablo Lopez-Bergami ${ }^{2, \ddagger}$, James S. Goydos ${ }^{3}$, Dana Yip ${ }^{3}$, Ameae M. \\ Walker $^{1}$, Elena B. Pasquale ${ }^{2,4}$, and Iryna Ethell ${ }^{1, \dagger}$ \\ ${ }^{1}$ Division of Biomedical Sciences, University of California Riverside, Riverside, CA, USA \\ ${ }^{2}$ Sanford-Burnham Medical Research Institute, La Jolla, CA, USA \\ ${ }^{3}$ Division of Surgical Oncology, University of Medicine and Dentistry of New Jersey, Robert Wood \\ Johnson Medical School, Piscataway, USA \\ ${ }^{4}$ Department of Pathology, University of California San Diego, CA, USA
}

\section{Keywords}

Eph receptor; ephrin; proliferation; Erk; Akt

Dear Sir,

Cutaneous melanoma is the most aggressive form of skin cancer and several families of receptor tyrosine kinases have been implicated in its development and progression, including the Eph receptor family (Hess et al., 2007; Smalley et al., 2009). Among Eph receptors, EphA2 has been most extensively studied in melanoma and linked to increased malignancy (Hess et al., 2007; Margaryan et al., 2009). The roles of other Eph receptors in melanoma progression, however, have not been extensively characterized. A recent study has shown that overexpression of EphB4 in murine B16 melanoma cells (which do not express the preferred EphB4 ligand, ephrin-B2) decreases the survival of ephrin-B2-positive tumor endothelial cells, suggesting that EphB4 may function as a tumor suppressor in melanoma by inhibiting angiogenesis (Huang et al., 2007). However, the widespread expression EphB4 in human cancers (Pasquale, 2010), including melanomas (Figure S1), suggests a possible positive role in tumor progression.

Our previous work showed that EphB4 promotes the migratory ability of a series of murine melanoma cell lines (Yang et al., 2006). SW1 and C19 represent distinctive clones derived from the same parental cell line. The more aggressive SW1 cells express high levels of EphB4 and have high migratory ability, whereas the less aggressive C19 cells have low EphB4 levels and migrate poorly. The two cell lines similarly express the ephrin-B2 ligand. We previously reported that EphB4 endogenously co-expressed with ephrin-B2 in SW1 cells or transiently transfected in $\mathrm{C} 19$ cells promotes cell migration in vitro by activating the RhoA GTPase, thus inducing actin cytoskeleton reorganization. Here we show that EphB4 co-expression with ephrin-B2 also promotes SW1 and C19 cell growth in vitro and tumor growth in vivo.

\footnotetext{
${ }^{\dagger}$ Correspondence: Nai-Ying Yang, Sanford-Burnham Medical Research Institute, 10901 N. Torrey Pines Rd., La Jolla, CA 92037; nyyang@burnham.org. Iryna Ethell, Division of Biomedical Sciences, University of California, Riverside, 900 University Avenue, Riverside, CA 92521-0121; iryna.ethell@ucr.edu.

*Current address: Instituto de Medicina y Biologia Experimental, Vuelta de Obligado 2490, Buenos Aires, Argentina
} 
We generated 4 stable C19 clones overexpressing EphB4 (C19-EphB4) as well as SW1 and C19 clones expressing EGFP (SW1 and C19; Figures 1A and S2A) and examined their proliferation by measuring BrdU incorporation and apoptosis by Hoechst nuclear staining. SW1 cells, which express high levels of endogenous EphB4, proliferate faster and exhibit less apoptosis compared to C19 cells. The C19-EphB4 clones, where the transfected EphB4 is expressed at levels similar to those in the SW1 clones, have proliferation and apoptotic rates comparable to the SW1 cells (Figures 1B,C and S2B,C). These results show that coexpression of EphB4 with ephrin-B2 promotes melanoma cell proliferation and survival in vitro. To investigate the in vivo role of EphB4 in melanoma growth, we used the stable clones to generate tumors in a mouse xenograft model. The SW1 and C19-EphB4 tumors grew faster than the $\mathrm{C} 19$ tumors over a period of 4.7 weeks, while there was no significant difference in body weight between the 3 groups of mice (Figure S2D). Enhancement of melanoma tumor growth by EphB4 was confirmed by injecting a mixture of 3 additional SW1, C19 or C19-EphB4 clones and measuring tumor growth for 7.5 weeks (Figure 1D). Several lung metastases were observed at 7.5 weeks in the mice bearing SW1 tumors, but not in the ones with either C19 or C19-EphB4 tumors (data not shown), suggesting that high EphB4 expression alone may not be sufficient to promote the formation of detectable metastases.

To investigate the signaling pathways that promote the growth of melanoma cells expressing high EphB4 levels, we first assessed EphB4 tyrosine phosphorylation as an indication of receptor activation. This revealed that EphB4 is substantially activated in the SW1 and C19EphB4 tumors (Figure 2A), consistent with the reported expression of the ephrin-B2 ligand in both SW1 and C19 cells and the extensive cell-cell contacts present in the 3-dimensional tumor environment (Yang et al., 2006). We then examined the effects of EphB4 expression on the activation of the Erk1/2 and the Akt kinases, which are known to play a critical role in melanoma cell transformation, survival and proliferation (Gray-Schopfer et al., 2007; Lopez-Bergami et al., 2008). We detected significantly higher levels of Erk1/2 phosphorylation at threonine 202 and tyrosine 204 and Akt phosphorylation at serine 473 in SW1 and C19-EphB4 tumors compared to C19 tumors, indicating increased activation of Erk1/2 and Akt (Figure 2B,C). We also detected higher levels of the anti-apoptotic protein Bcl-2 in the SW1 and C19-EphB4 tumors than the C19 tumors (Figure 2D). The higher Erk1/2 and Akt activity (which are consistent with the EphB4-dependent activation of Akt previously observed in breast cancer and endothelial cells (Steinle et al., 2002; Kumar et al., 2006)) and the higher Bcl-2 expression, would all be expected to contribute to the faster growth of tumors expressing activated EphB4.

Besides cell proliferation and apoptosis, angiogenesis is another important event that contributes to tumor progression. Given that EphB4 can activate reverse signaling through ephrin-B2 on adjacent endothelial cells to promote angiogenesis and blood vessel remodeling (Pasquale, 2010), we also examined the effects of EphB4 on tumor vascularization. Quantitative analysis of CD31-stained tumor sections revealed that the blood vessels were significantly larger in both SW1 and C19-EphB4 tumors than in C19 tumors at both 4.7 weeks and 7.5 weeks (Figures 2E and S3A). No significant difference was observed in blood vessel densities between the 3 groups, although more blood vessels were formed over the same time period in the tumors expressing EphB4, given the larger size of these tumors. To verify that the vascular differences observed were not due to the different tumor sizes, we also examined tumors of similar size, which were collected at different times after melanoma cell injection. SW1 and C19-EphB4 tumors similar in size to the C19 tumors also had larger blood vessels (Fig. S3B). These data suggest that EphB4 expression causes blood vessel growth and enlargement in SW1 and C19-EphB4 melanoma tumors. If increased vascularization in EphB4-expressing tumors supports faster growth of 
tumor cells, this would result in larger tumors with similar vascular densities (Kerbel and Folkman, 2002).

Although EphB4 promotes SW1 and C19 melanoma cell malignancy, this receptor has been reported to suppress tumorigenicity in breast and colorectal cancer cells (Pasquale, 2010). These divergent activities may depend in part on whether the ephrin-B2 ligand is coexpressed and persistently activates the receptor and on other contextual factors. In addition, ephrin-B2 can also transduce signals through its cytoplasmic domain, which are known as reverse signals and are triggered by binding to Eph receptors (Pasquale, 2010; Meyer et al., 2005). Since both EphB4 and ephrin-B2 are present in SW1 and C19-EphB4 cells, we cannot exclude that some of the tumor promoting effects observed could be due to ephrin$\mathrm{B} 2$ reverse signaling.

In conclusion, we show that besides promoting RhoA-dependent migration (Yang et al., 2006), EphB4 can promote the growth of melanomas expressing the ephrin-B2 ligand by stimulating proliferation, survival and angiogenesis. Our findings suggest that upregulation of EphB4 receptor expression can play a role in melanoma progression, particularly in tumors where Erk and/or Akt are not highly activated by mutations.

\section{Supplementary Material}

Refer to Web version on PubMed Central for supplementary material.

\section{Acknowledgments}

The authors thank Z. Ronai for helpful discussion and comments on the manuscript and N. K. Noren for advice regarding the immunoblots of human melanoma specimens. This work was supported by a University of California Cancer Research Coordinating Committee grant (IME), NIH grant CA116099 (EBP), and DOD postdoctoral fellowship W81XWH-09-1-0665 (NYY).

\section{References}

Gray-Schopfer V, Wellbrock C, Marais R. Melanoma biology and new targeted therapy. Nature. 2007; 445:851-857. [PubMed: 17314971]

Hess AR, Margaryan NV, Seftor EA, Hendrix MJ. Deciphering the signaling events that promote melanoma tumor cell vasculogenic mimicry and their link to embryonic vasculogenesis: Role of the Eph receptors. Dev Dyn. 2007; 236:3283-3296. [PubMed: 17557303]

Huang X, Yamada Y, Kidoya H, Naito H, Nagahama Y, Kong L, Katoh SY, Li WL, Ueno M, Takakura N. EphB4 overexpression in B16 melanoma cells affects arterial-venous patterning in tumor angiogenesis. Cancer Res. 2007; 67:9800-9808. [PubMed: 17942910]

Kerbel R, Folkman J. Clinical translation of angiogenesis inhibitors. Nat Rev Cancer. 2002; 2:727739. [PubMed: 12360276]

Kumar SR, Singh J, Xia G, Krasnoperov V, Hassanieh L, Ley EJ, Scehnet J, Kumar NG, Hawes D, Press MF, et al. Receptor tyrosine kinase EphB4 is a survival factor in breast cancer. Am J Pathol. 2006; 169:279-293. [PubMed: 16816380]

Lopez-Bergami P, Fitchman B, Ronai Z. Understanding signaling cascades in melanoma. Photochem Photobiol. 2008; 84:289-306. [PubMed: 18086245]

Margaryan NV, Strizzi L, Abbott DE, Seftor EA, Rao MS, Hendrix MJ, Hess AR. EphA2 as a promoter of melanoma tumorigenicity. Cancer Biol Ther. 2009; 8:279-288. [PubMed: 19223760]

Meyer S, Hafner C, Guba M, Flegel S, Geissler EK, Becker B, Koehl GE, Orso E, Landthaler M, Vogt T. Ephrin-B2 overexpression enhances integrin-mediated ECM-attachment and migration of B16 melanoma cells. Int J Oncol. 2005; 27:1197-1206. [PubMed: 16211213]

Pasquale EB. Eph receptors and ephrins in cancer: bidirectional signalling and beyond. Nat Rev Cancer. 2010; 10:165-180. [PubMed: 20179713] 
Smalley KS, Sondak VK, Weber JS. c-KIT signaling as the driving oncogenic event in sub-groups of melanomas. Histol Histopathol. 2009; 24:643-650. [PubMed: 19283671]

Steinle JJ, Meininger CJ, Forough R, Wu G, Wu MH, Granger HJ. Eph B4 receptor signaling mediates endothelial cell migration and proliferation via the phosphatidylinositol 3-kinase pathway. J Biol Chem. 2002; 277:43830-43835. [PubMed: 12235151]

Yang NY, Pasquale EB, Owen LB, Ethell IM. The EphB4 Receptor-tyrosine Kinase Promotes the Migration of Melanoma Cells through Rho-mediated Actin Cytoskeleton Reorganization. J Biol Chem. 2006; 281:32574-32586. [PubMed: 16950769] 


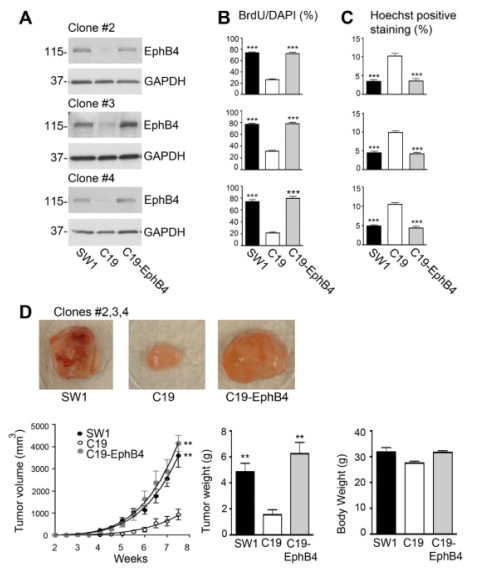

Figure 1.

EphB4 promotes melanoma cell proliferation and inhibits apoptosis in vitro and promotes melanoma tumor growth in vivo. (A) EphB4 expression levels in 3 SW1, C19 and C19EphB4 clones (data for clone \#1 are shown in Figure S2). Lysates were probed by immunoblotting for EphB4, and GAPDH as a loading control. (B) Cell proliferation assessed by measuring BrdU incorporation. The histograms show average percentages of BrdU-positive cells 2 hours following BrdU application \pm SEM ( $n \sim 1,000$ cells per group from 3 independent experiments; ***p $<0.001$ compared with the $\mathrm{C} 19$ cells by one-way ANOVA). (C) Apoptosis assessed by Hoechst 33342 staining. The histograms show average percentages of Hoechst-positive cells \pm SEM ( $n \sim 2,250$ cells per group from 3 independent experiments; *** $<0.001$ compared with the $\mathrm{C} 19$ cells by one-way ANOVA). (D) Photographs of representative 7.5 week-old tumors derived from a mixture of 3 clones (\#2, $\# 3$ and \#4) of SW1, C19 or C19-EphB4 cells. The growth curves show average tumor volumes \pm SEM measured at the indicated times in groups of 6 mice. ${ }^{* *} p<0.01$ compared with the $\mathrm{C} 19$ group using repeated measures two-way ANOVA. The histograms show average tumor weights and body weights \pm SEM measured at 7.5 weeks $(n=6$ tumors per group; $* * p<0.01$ compared with the $\mathrm{C} 19$ group by one-way ANOVA). 


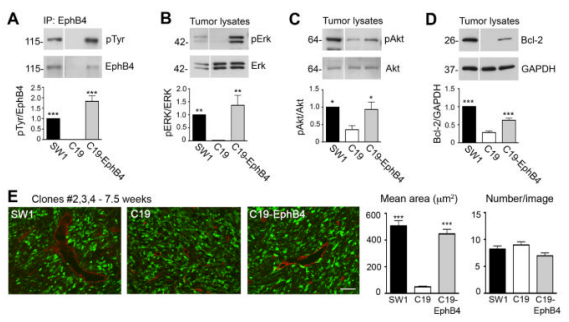

Figure 2.

EphB4 upregulates Erk and Akt phosphorylation and Bcl-2 expression, and promotes blood vessel enlargement in melanoma tumors. (A) EphB4 immunoprecipitates from tumors grown for 7.5 weeks were probed for phosphotyrosine (pTyr) and reprobed for EphB4. The histogram shows average levels of phosphorylated EphB4 quantified by densitometry and normalized to total EphB4 \pm SEM ( $n=4$ tumors per group; $* * * p<0.001$ compared with the C19 tumors by one-way ANOVA). (B, C, D) Tumor lysates were probed for phosphoErk1/2 Thr202/Tyr204 and total Erk1/2, phosphoAkt S473 and total Akt, Bcl-2 and GAPDH. The histograms show the average levels of pErk, pAkt or Bcl-2 quantified by densitometry and normalized to total Erk, total Akt or GAPDH \pm SEM ( $n=4$ tumors per group; * $p<0.05$; $* * \mathrm{p}<0.01 ; * * * \mathrm{p}<0.001$ compared with the C19 tumors by one-way ANOVA). The lanes in each blot are from the same gel and the white space indicates that an irrelevant lane between the SW1 and C19 lanes was removed. (E) Fluorescent images of EGFP-positive melanoma cells (green) and blood vessels stained with anti-CD31 antibody (red) in frozen sections from tumors collected at 7.5 weeks. The histograms show average areas occupied by blood vessels and number of blood vessels per image (image area $180 \mathrm{~mm}^{2}$ ). Scale bar, 2 $\mu \mathrm{m}$. The histograms show averages \pm SEM $(\mathrm{n}=16$ sections from $3-4$ mice corresponding to $\sim 1,000$ blood vessels per group; $* \mathrm{p}<0.05 ; * * \mathrm{p}<0.01$; *** $\mathrm{p}<0.001$ compared to $\mathrm{C} 19$ tumors by one-way ANOVA). 